\title{
Team ARAIBO
}

Yuichi KOBAYASHI and Hideo YUASA

The University of Tokyo

\section{Introduction}

Our team focused on manipulating the ball. We developed the kicking and heading motion. Two kinds of neural networks are utilized in order to recognize the ball and kick it. The recognition and manipulation did not work sufficiently, but intdicates our interest of playing soccer with legged robots. We confronted the team LRP and the team McGill in the round robin. We defeated McGill by PK, and lost the game with LRP 0-2.

\section{Team Development}

Team Leader: Tamio ARAI

Team Members: Tamio ARAI

- Dept. of Precision Machinery Engineering, The University of Tokyo

- Japan

- Professor

- did not attend the competition

Hideo YUASA

- Dept. of Precision Machinery Engineering, The University of Tokyo

- Japan

- Professor

- attended the competition

Yuichi KOBAYASHI

- Dept. of Precision Machinery Engineering, The University of Tokyo

- Japan

- Student in the doctor course

- attended the competition

Masaki Fukuchi

- Dept. of Precision Machinery Engineering, The University of Tokyo

- Japan

- Student in the master course

- attended the competition

Jun'ichi IMANISHI

- Dept. of Precision Machinery Engineering, The University of Tokyo

- Japan

- Student in the master course

- attended the competition

Toru IWATA

M. Veloso, E. Pagello, and H. Kitano (Eds.): RoboCup-99, LNAI 1856, pp. 758-761, 2000.

(C) Springer-Verlag Berlin Heidelberg 2000 
- Dept. of Precision Machinery Engineering, The University of Tokyo

- Japan

- Student in the master course

- attended the competition

Web page http://www.arai.pe.u-tokyo.ac.jp/robocup

\section{Complete Robot Architecture}

We did not integrate reflex hehaviors such as avoiding obstacles or other exceptional behaviors. So the robots decided actions based on the recognition of the ball and the information of self-localization.

When the robot does not recognize the ball, it continues to turn at the same place with its head swinging. In order to avoid dead-rock state, it keeps the time since the current walking command has been decided. If the walking action does not change over a threshold time value, it changes its walking action by a random value unconditionally.

\section{Vision}

The 8 channels of the color detector are set, orange, green, white, skyblue, yellow, pink, darkblue and red. Darkblue and red are the colors of robots. They are recognized by the color detector but are not used for action dicision. When the color pink is detected, upper and lower regions of the center of pink are referenced. The type of the landmark is decided by comparing the number of pixels of each color. The goals are recognized when skyblue and yellow are detected without pink.

We made the color table as follows:

- Take images and save in memory sticks.

- Create image file in the 'ppm' format.

- Pick up the region where we want to recognize.

- Make a ppm image file that consists of black and white. Black corresponds to the color we want to recognize, and white other colors.

- Pick up YUV values of each pixel which correspond to a black pixel.

- Decide max and minimum YUV values of the color by collecting data.

In order to measure the ball position more precisely, we used Back Propagation algorithm. The ordinal way of recognizing the ball position is to utilize the pan and tilt joint values of the head while the head is tracking the ball. This way is not so much reliable especially when the ball comes near to the body. We developed the way of refining the measurement by utilizing the four parameters of the ball image.

First, we collect image data of the ball at a fixed position changing the pan and tilt joint angles of the head. The pan and tilt joint angles are not always the same even when the position of the ball is the same. The collected data consist 
of pan and tilt joint angle values and four parameters mentioned above. These six variables are measured mostly around ten times with different pan and tilt angles.

The first neural network is used to calculate the ideal pan and tilt joint. Here the word 'ideal joint' means that the CCD camera captures the ball exactly in the center of the visual field with the pan and tilt joint angles. In such a state, these angles provides enough information to specify the position of the ball. The stored data are used to solve the inverse problem. The training data of the network consist of $\mathrm{x}$ and $\mathrm{y}$ value of the center of the ball in the visual field as inputs and the pan and tilt joint values as outputs. After training, the ideal pan and tilt joint value is calculated by giving this network the center of the visual field.

Then the position of the ball is changed and fixed. The same process is iterated by changing the ball position. The second neural network is used to express the relation between six parameters and ideal pan and tilt joint values. Input data consists of actual pan and tilt joint values and four image parameters. Output data consists of ideal pan and tilt joint values which are calculated by the first neural network. By training with the data set of them, ideal pan and tilt joint values can be calculated from the actual joint values and image parameters.

\section{Control}

The manipulation actions for the ball are kicking and heading. When the robot kicks the ball keeping the posture of standing, the ball does not go so far because the reach of the kicking leg is limited. So, we gave up preserving the standing posture during the kicking motion. First, the robot bends its right ( or left ) front leg. The robot begins to fall down, and then the robot extends its bent leg. The ball gets farthest when the extending action sinchronizes with the fall of the body.

When the goal is the side of the robot, it hits the ball by its head. Two motions are combined to realize this heading action. One is the swinging motion of the head, and the other is the translation of the body by the legs. shows the motion of legs to translate the body. The heading action is achieved by the conbination of this action and the swinging action of the head.

\section{Localization}

We used roughly estimating algorithm from only one landmark. When one landmark is recognized, the current direction of the robot can be estimated very roughly.

When the position and posture of the robot is estimated in some way, these variables are transformed to the relative relation between the robot and the goal. The robot can update these relative relations by dead reckoning. 


\section{Strategy}

The main strategy of our robots is to 'find the ball, and get close to the ball', and to 'manipulate the ball according to the direction of the goal'. The two modes ball-searching and ball-tracking correspond to the most basic condition.

When the mode is ball-tracking, the robot approaches to the ball and decides how to manipulate the ball. Here we mean kicking and heading action for the word 'manipualte'. The robot changes walking command so as to adjust the pan angle of the head to zero.

If the pixel number of the orange ball exceeds a threshold value, it decides the manipulation action according to the roughly estimated direction of the goal.

When the mode is ball-searching, the robot turns its body while swinging its head. In order to avoid dead-rock state, it changes the walking action when the same walking action continues over a threshold time.

\section{Special Team Features}

We developed learning system to realize 'Kick the ball where the robot wants to'. This architecture is based on Kohonen's Self-Organizing Maps(SOMs). Here we used SOM as associative memory. Action and its evaluation data is stored beforehand by the experiment. The weight vector of each node consist of as state inputs, as action outputs and as evaluation signals. Here, the state inputs are the ideal pan and tilt joint angle values calculated by the neural network. The action outputs are the direction of the kicking leg. The evaluation signals are the direction and distance of the ball after kicking. The distance is estimated by the number of pixels in the visual field.

The best matching node is calculated with the state inputs and desired evaluation signals. Action outputs and estimation of evaluation signals are decided by the best matching node. Actually the difference of the action output is not so influential with the direction of the ball. At least, this stored data can be utilized to estimate the direction of the ball before kicking. When the robot knows that the ball doesn't go to the disired direction with any action output, it can change the position by walking.

But this learning architecture did not work sufficiently in Stockholm. This is partly because the calculation of the ball position mentioned above is not precise enough.

\section{Conclusion}

We are going to participate the league next year. Development of the ability of ball operation is indispensable in order to realize more complicated strategy. We should focus on recognition and manipulation of the ball in front of the body , continuously. Learning for acquisition of the relation between manipulation and observation will be the main problem of our approach.

This article was processed using the $\mathrm{LT}_{\mathrm{E}} \mathrm{X}$ macro package with LLNCS style 